\title{
Association of two apolipoprotein A-I gene MspI polymorphisms with lipid and blood pressure levels
}

Yan Qing Ma, PhD; G Neil Thomas, PhD*; Brian Tomlinson, FRCP, MD

Department of Medicine and Therapeutics, The Chinese University of Hong Kong, Prince of Wales Hospital, Shatin, Hong Kong. *Department of Community Medicine, University of Hong Kong, Hong Kong

Short title: Ma, Thomas \& Tomlinson: ApoA-I/MspI polymorphisms in Chinese

This study was supported by a Direct Grant for Research (code 2040526) from the Medicine Panel, The Chinese University of Hong Kong and grants from Hong Kong Research Grants Council (grants CUHK 4095/00M).

*all correspondence:

G Neil Thomas

Department of Community Medicine

5/F Academic and Administration Block

Faculty of Medicine Building

The University of Hong Kong

21 Sassoon Road, Hong Kong

Tel: (852) 2819 9878; Fax: (852) 28559528

E-mail: gneilthomas@yahoo.co.uk 


\begin{abstract}
Introduction: Two MspI polymorphisms in the ApoA-I gene (G-75A and C83T) have been shown to be associated with plasma HDL-cholesterol levels.

Methods: We used a PCR-based RFLP method to determine the association of these polymorphisms with lipid parameters in 271 non-diabetic, normotriglyceridaemic Chinese subjects, of whom 104 were patients with hypertension, with $10.2 \%$ having hypercholesterolaemia and the remainder were controls.
\end{abstract}

Results: As expected, the hypertensive group had higher blood pressure and indices of obesity, and a more adverse lipid profile. No differences in the ApoA-I G-75A genotype or allele frequency distributions between the controls and patients were identified. However, there was a significantly lower frequency of the CT genotype $(\mathrm{p}=0.012)$ and $\mathrm{T}$ allele $(p=0.011)$ in the affected subjects with hypercholesterolaemia or hypertension. Similarly, blood pressure and triglyceride levels were significantly lower and HDL-cholesterol levels significantly higher in the subjects with the CT genotype compared to those with the CC genotype ( $<$ 0.05). However, the G-75A genotypes did not appear to influence the lipid or blood pressure levels. The -75A allele frequency was higher in our healthy controls than an equivalent Caucasian population $(31.1 \%$ vs. $18.3 \%, \mathrm{p}<0.001)$, whereas the $83 \mathrm{~T}$ allele frequency was similar between the healthy Chinese and Caucasian groups.

Conclusion: The 83T allele may be associated with a better lipid profile and blood pressure levels in this group of Chinese subjects.

Key words: Apolipoprotein A-I, Chinese, hypertension, lipids, metabolic syndrome, polymorphisms 


\section{Introduction}

Apolipoprotein A-I (apoA-I) is the major structural protein of high density lipoprotein (HDL) particles and, as a co-factor of lecithin-cholesterol acyltransferase, is involved in the esterification of free cholesterol [1,2]. ApoA-I plays an essential role in cholesterol efflux from peripheral cells and in the reverse cholesterol transport process. Several epidemiological studies have reported that HDL-cholesterol levels are inversely related to cardiovascular disease including the incidence of coronary heart disease (CHD) [3-5]. Weekly infusions of apoA-I Milano, a mutant version of apoA-I found in subjects with low HDL-cholesterol, but not increased risk of vascular disease, for 5 weeks was found to regress, coronary atheroma volume in patients hospitalised with acute coronary syndromes [6]. These studies further support a role for apoA-I and HDL-cholesterol in the pathogenesis of cardiovascular disease.

The regulation of HDL levels is a complicated and incompletely understood process, and is likely to depend on the interaction of both environmental and genetic factors. Several genetically determined structural variants of apoA-I have been identified, and have allowed the characterisation of structure-function relationships in the protein [7].

A $G \rightarrow$ A mutation at position -75 of the apoA-I gene promoter occurs at a frequency of about 0.18 in Caucasian populations and has been associated with higher plasma HDLcholesterol levels in several ethnic groups [8-17]. In vitro [18] and in vivo studies [15-17] have shown that the A substitution is associated with increased apoA-I gene expression, and elevated plasma apoA-I and HDL-cholesterol concentrations. Up-regulation of the apoA-I promoter may lead to increased HDL production and increased very low- and low-density lipoprotein (LDL) clearance. Previous studies also showed an association between the apoAI polymorphism and plasma triglyceride levels, the triglyceride level being lower in men with $-75 \mathrm{~A}[14,19]$. 
A further MspI polymorphic site has been identified in the first intron of the apoA-I gene [20], in which two consecutive transitions at +83 bp ( $\mathrm{C}$ to $\mathrm{T}$ ) and +84 bp ( $\mathrm{G}$ to A) sites occur together or independently. Several studies have shown that these transitions are also associated with increased HDL-cholesterol levels in Caucasians [21]. The frequency of the +83 bp substitution in a healthy Caucasian population was lower (rare allele frequency: 0.04) than that at the -75 bp site [21]. However, some studies reported that patients with the base changes at -75 and/or +83 bp of the apoA-I gene have more severe CHD in both Caucasian and Chinese populations [21,22]. In those studies, base changes at -75 and +83 bp of the apoA-I gene were not associated with increased HDL-cholesterol levels.

In the present study we investigated the two MspI polymorphisms (G-75A and C83T) to assess their importance, with respect to plasma lipid levels and blood pressure in Chinese subjects with hypertension and non-hypertensive controls.

\section{Methods}

The study was approved by the Clinical Research Ethics Committee of the Chinese University of Hong Kong. All 271 subjects gave written informed consent and were of Han Chinese origin, without any known ancestors of other ethnic origin, and were living in the Hong Kong Special Administrative Region of China at the time of the study. Patients ( $n=104$, group A) with hypertension, of whom 10.2\% had hypercholesterolaemia, were recruited from the general outpatient clinics at the Prince of Wales Hospital. The catchment area of the Prince of Wales Hospital has only been developed since the 1960's, and serves a population of over 1 million. The majority of its inhabitants, including staff at the hospital, are a typical socio-economic representation of first or second-generation migrants from Southern China now living in a Westernised environment. The Prince of Wales Hospital is a teaching hospital and tertiary referral centre. However, as in other public hospitals in Hong 
Kong, because the system of government-funded primary care is not developed to the same extent as most Western countries, many of the patients attending the clinics use the facility as their only source of subsidised medical care for management of their chronic diseases. The patients involved therefore do not represent a highly selected group of the most severe cases, but are a typical cross-section of patients with these conditions from this region of Hong Kong. Subjects seen by the study physicians in the medical outpatient clinics at the Prince of Wales Hospital meeting the selection criteria described below were consecutively invited to participate in the study. All subjects were screened between 9:00 and 10:30 AM and treatments for hypertension or dyslipidaemia were withheld until after the measurement of blood pressure and the collection of blood samples, following an overnight fast. Healthy controls (group B) were recruited from a broad spectrum of hospital staff. Subjects were defined as hypercholesterolaemic if their total cholesterol level was $\geq 6.2 \mathrm{mmol} / \mathrm{L}$ or total cholesterol level was $<6.2 \mathrm{mmol} / \mathrm{L}$, but $\geq 5.2 \mathrm{mmol} / \mathrm{L}$ and total cholesterol to HDLcholesterol ratio was $\geq 5.0$ or were on treatment to lower LDL-cholesterol. Subjects with more severe hypertriglyceridaemia $(\geq 2.3 \mathrm{mmol} / \mathrm{L}$ ) were excluded as it was considered that they were likely to have other genetic causes and the close inverse relationship between triglycerides and HDL-cholesterol would mask any relationship between the ApoA-I gene polymorphisms and lipid levels or blood pressure. Subjects were defined as hypertensive if seated systolic blood pressure was $\geq 140 \mathrm{~mm} \mathrm{Hg}$ and/or diastolic blood pressure was $\geq 90 \mathrm{~mm}$ Hg after repeated measurement in a quiet environment or were receiving anti-hypertensive treatment. Only $10 \%$ of the hypertensive subjects were receiving treatment. All subjects with impaired fasting glucose or diabetes (fasting plasma glucose $\geq 6.1 \mathrm{mmol} / \mathrm{L}$ ) were excluded.

DNA was extracted from peripheral blood from the 271 Hong Kong Chinese subjects. The apoA-I gene C83T and G-75A polymorphisms were screened using a PCR-based RFLP protocol [14]. The presence of the MspI restriction sites at $-75 \mathrm{bp}$ ( $\mathrm{G}$ allele) and at $+83 \mathrm{bp}(\mathrm{C}$ 
allele) in the 433 bp product resulted in four fragments of 209 bp, 113 bp, 66 bp and 45 bp. The absence of the restriction site at -75 bp (A allele) resulted in three fragments of 209 bp, 179 bp and 45 bp. The absence of the restriction site at +83 bp ( $\mathrm{T}$ allele) created a larger fragment of 254 bp instead of two fragments of 209 bp and 45 bp.

Statistics Package for the Social Science (SPSS version 11.0, 2002, SPSS Inc, Chicago, II) was used in the data analyses. The frequencies of the alleles and genotypes among the control and hypertensive groups ( $\mathrm{A}$ and $\mathrm{B}$, respectively) were compared by the chi-square test. The independent t test was used to examine the association between the alleles and clinical parameters. For parameters with skewed distributions, the nonparametric Mann-Whitney test was performed. An analysis of variance (ANOVA) was performed to examine the contribution of the G-75A and C83T polymorphisms on plasma lipid levels and blood pressure.

Gender was coded 0 and 1 for male and female, respectively. For the genetic parameters, the apoA-I G-75A GG, GA and AA genotypes were coded 0, 1, and 2, and; C83T CC, CT genotypes were coded 0 and 1 . The variables included in the analyses were linearly related to the dependent variables. Zero order and partial correlations analyses adjusted for age, and/or gender, were performed to investigate for relationships between the blood pressure and lipid parameters and the apoA-I genotypes. For the stepwise multiple regression to determine independent predictors of systolic and diastolic blood pressures and HDLcholesterol, age, gender, smoking, systolic and diastolic blood pressures, waist-to-hip ratio, body mass index, LDL and HDL-cholesterol and triglycerides, glucose, insulin-glucose product, and the apoA-I polymorphism genotypes were included in the analyses. Due to a close correlation, triglycerides were excluded in the determination of independent predictors of HDL-cholesterol, and only either systolic or diastolic blood pressures was included during the determination of predictors of blood pressure. The appropriateness of the regression 
model was judged from the Durbin-Watson statistic (testing for serial correlation of adjacent error terms), and partial plots of the residuals. The tolerance and variance inflation factors (VIF) were taken as measures of collinearity, with low tolerance and high VIF being signs of collinearity indicating that a variable should not be included in the model.

\section{Results}

A total of 271 non-diabetic, normotriglyceridaemic Chinese subjects were recruited for the study. The demographic characteristics of the control and hypertensive subjects are described in Table 1. The hypertensive group (B) not only had higher blood pressure but also higher indices of obesity, and a more adverse lipid profile than the control group A.

No differences were identified in the frequency distributions of the ApoA-I G-75A genotypes or alleles between the control (group A) and hypertensive subjects (group B). However, there was a significantly lower frequency of the CT genotype $(p=0.012)$ and $T$ allele $(\mathrm{p}=0.011$ ) in the hypertensive subjects (group B). Similarly, blood pressure and triglyceride levels were significantly lower and HDL-cholesterol was significantly higher in the subjects with the CT genotype compared to those with the CC genotype ( $\mathrm{p}<0.05$ for all, Table 2). However, the G-75A genotypes did not appear to be associated with the lipid or blood pressure levels.

From the zero order correlation analyses, the apoA-I C83T polymorphism correlated with HDL-cholesterol $(\beta=0.14, p=0.029)$, triglycerides $(\beta=-0.17, p=0.006)$, systolic $(\beta=-0.15$, $\mathrm{p}=0.018)$ and diastolic blood pressure $(\beta=-0.12, \mathrm{p}=0.056)$. Partial correlation adjustment for gender had no effect on the strength of the correlation, but after adjustment for age and gender the relationship with systolic blood pressure was no longer significant $(\beta=-0.10$, $\mathrm{p}=0.12$ ), but remained significant for HDL-cholesterol $(\beta=0.13, \mathrm{p}=0.047)$, and triglycerides $(\beta=-0.12, p=0.043)$. 
Using stepwise multiple linear regression, waist circumference $(\beta=-0.32, p<0.001)$ and gender ( $\beta=0.18, \mathrm{p}=0.033$ ) were identified as independent predictors of HDL-cholesterol, accounting for $18 \%$ of the variance $(\mathrm{HDL}$-cholesterol $=[-0.01 \cdot$ waist circumference $]+[0$. 41.female gender $\left.]+2.2, \mathrm{R}^{2}=0.18, \mathrm{~F}=15.4, \mathrm{p}<0.001\right)$. For triglyceride levels, systolic blood pressure $(\beta=0.21, p=0.012)$, waist circumference $(\beta=0.24, p=0.011)$, fasting insulin-glucose product $(\beta=0.19, \mathrm{p}=0.011)$ and age $(\beta=0.17, \mathrm{p}=0.021)$ were identified as independent predictors of triglycerides, accounting for $40 \%$ of the variance (triglycerides $=[0.02 \cdot$ systolic blood pressure $]+[0.05$ waist circumference $]+[0.15$ fasting insulin-glucose product $]+$ [0.003.age] - 1.0, $\left.\mathrm{R}^{2}=0.40, \mathrm{~F}=23.9, \mathrm{p}<0.001\right)$. For systolic blood pressure, waist circumference $(\beta=0.52, \quad \mathrm{p}<0.001)$, triglycerides $(\beta=0.21, \mathrm{p}=0.003)$, and age $(\beta=0.16$, $\mathrm{p}=0.015$ ) were independent predictors, accounting for $54 \%$ of the variance (systolic blood pressure $=[1.18 \cdot$ waist circumference $]+[22.7 \cdot$ triglycerides $]+[0.33 \cdot$ age $]+28.5, \mathrm{R}^{2}=0.54$, $\mathrm{F}=56.4, \mathrm{p}<0.001)$. For diastolic blood pressure, the same parameters waist circumference $(\beta=0.53, \mathrm{p}<0.001)$, age $(\beta=0.17, \mathrm{p}=0.010)$, and triglycerides $(\beta=0.18, \mathrm{p}=0.015)$ were independent predictors, accounting for $53 \%$ of the variance (diastolic blood pressure $=$ [0.82.waist circumference $]+[0.24 \cdot$ age $]+[13.0$-triglycerides $]+5.3, \mathrm{R}^{2}=0.53, \mathrm{~F}=52.7$, $\mathrm{p}<0.001)$

Data previously reported from Caucasian subjects ( $n=534)$, where the frequency of the GG, GA, and AA genotypes were 65.7, 32.0, and 2.3\%, respectively, with an allele frequency of 81.7 , and $18.3 \%$, for the $\mathrm{G}$ and $\mathrm{A}$ alleles, were significantly different compared to the distribution within the current control population (group A, Table 2, p<0.001) [12]. Whereas no differences were observed for the C83T polymorphism, whose frequencies in Caucasians were 93.4, 6.6\% for the CC and CT genotypes and 96.7, 3.3\% for the C and T alleles [12]. 


\section{Discussion}

The prevalence of hypertension and dyslipidaemia is high in Hong Kong. In the adult population of Hong Kong Chinese, $46 \%$ of men and $41 \%$ of women have plasma cholesterol levels over $5.2 \mathrm{mmol} / \mathrm{L}$. $18 \%$ of men and $17 \%$ of women have hypertension $(\geq 140 / 90 \mathrm{~mm}$ Hg) [23]. Many genetic and environmental factors are involved in regulation of lipid levels and blood pressure. The apoA-I gene, which is a major structural component of HDL, is a possible candidate associated with changes in lipid metabolism. Epidemiological and clinical studies have revealed an inverse correlation between plasma HDL-cholesterol concentration and CHD incidence [24-26]. Furthermore, a strong genetic regulation of the level of HDLcholesterol has been established in several studies [25,27]. In an effort to elucidate the functional role of apoA-I gene polymorphisms, we examined a putative association between two apoA-I/MspI polymorphisms and plasma lipid levels, and explored their relationship with other clinical parameters.

In vitro and in vivo studies suggest that the A allele at -75 site increases apoA-I gene expression and hence leads to elevated plasma apoA-I and HDL-cholesterol concentrations [14-16,18,28]. Conversely, the A allele has also been reported to be associated with decreased apoA-I gene expression in vitro [29]. The base substitution at the 83 bp site of the apoA-I gene has also been reported to be associated with elevated HDL-cholesterol levels [21]. In our present study, the 83T allele was associated with higher HDL-cholesterol levels, supporting the association of this gene with modulating HDL-cholesterol levels, even though there was no evidence of a relationship with the G-75A polymorphism. The apoA-I C83T polymorphism was not an independent predictor of HDL-cholesterol levels. However, regression equations are designed to explain the largest proportion of the variance in the model, rather than identifying biologically relevant parameters involved in disease 
pathogenesis. As such, waist circumference, an independent predictor of all the lipid and blood pressure parameters, gender, or age may act as composite markers of a number of cardiovascular risk factors, and therefore appear as strong independent predictors in these analyses. Similarly, exclusion of a parameter does not preclude it from directly contributing to the pathogenesis of the disorder, merely the variance attributed to the parameter is accounted for by the other variables. In our cross-sectional analyses, BMI closely correlated with all blood pressure and lipid parameters, but was not an independent predictor in the regression analyses, probably confounded by other parameters such as increasing waist circumference and ageing. It is therefore not surprising that the apoA-I C83T polymorphism with its small effect size, and the likely interactions with other genetic and environmental factors, is not an independent predictor of HDL-cholesterol levels, even though the polymorphism correlated with HDL-cholesterol and triglyceride levels even after adjustment for age and gender.

The previous studies reported that the frequency of the $-75 \mathrm{~A}$ allele and the $+83 \mathrm{~T}$ in Caucasian populations is about $18 \%$ and $4 \%$, respectively $[8,12,15,16,21]$. As a distinct group, the Hong Kong Chinese healthy controls (group A) had a significantly higher -75A allele frequency (31.1\%) than in equivalent Caucasian populations. This suggests the genetic background could be responsible for the variable results.

Plasma triglyceride levels were significantly different between the C83T genotypes, showing decreased levels in the heterozygote subjects. Thus, the rare $\mathrm{T}$ allele was associated with lower plasma triglyceride levels. Mutations in the apoAI-CIII-AIV gene cluster have been implicated in hypertriglyceridaemia. The A allele at -75 bp site of apoA-I gene was associated with lower triglyceride levels in men in Finnish population [14], but not in Japanese [10] or in the current study. However, the association of the 83T allele with 
decreased triglyceride levels suggests this locus may modulate triglyceride levels in Oriental subjects.

Our study also found a relationship between the C83T polymorphism of the apoA-I gene and blood pressure. The $\mathrm{T}$ allele frequency was significantly lower in the affected subjects (group B) than that in control subjects (group A) and the subjects with the CT genotype had significantly lower SBP and DBP than subjects with CC genotype. Blood pressure is influenced by genetic and nongenetic factors. Other candidate genes in lipoprotein metabolism have been suggested to be candidates for blood pressure variation including the apoB gene which was reportedly associated with SBP [30]. The D8S282 marker near LPL gene locus has also been reported to be associated with SBP variation in Taiwanese [31] and in our population [32]. Furthermore, a previous study in Caucasians also suggested a possible role for the apoAI-CIIII-AIV gene complex in blood pressure [33]. Our findings support the close relationship between blood pressure and lipid metabolism. However, it is currently unclear how such a mutation might influence blood pressure but these polymorphisms might be in linkage with others which may be more clearly related to blood pressure.

In summary, we conclude that the allele distribution of the ApoA-I/MspI G-75A polymorphism is significantly different between Chinese and Caucasian populations. The G75A polymorphism did not appear to be associated with plasma HDL-cholesterol levels in Hong Kong Chinese. However, the 83T allele appears to be associated with higher HDLcholesterol levels and decreased triglyceride levels and blood pressure. The interesting situation with the apoA-I gene in linking lipid metabolism and blood pressure requires further investigation to elucidate the mechanisms underlying this relationship. 


\section{References}

1. Francone OL, Gurakar A, Fielding C. Distribution and functions of lecithin:cholesterol acyltransferase and cholesteryl ester transfer protein in plasma lipoproteins. Evidence for a functional unit containing these activities together with apolipoproteins A-I and D that catalyzes the esterification and transfer of cell-derived cholesterol. J Biol Chem 1989;264:7066-7072.

2. Francone OL, Royer L, Haghpassand M. Increased prebeta-HDL levels, cholesterol efflux, and LCAT-mediated esterification in mice expressing the human cholesteryl ester transfer protein (CETP) and human apolipoprotein A-I (apoA-I) transgenes. J Lipid Res $1996 ; 37: 1268-1277$.

3. Barter PJ, Rye KA. High density lipoproteins and coronary heart disease. Atherosclerosis 1996;121:1-12.

4. Hausmann D, Johnson JA, Sudhir K, et al. Angiographically silent atherosclerosis detected by intravascular ultrasound in patients with familial hypercholesterolaemia and familial combined hyperlipidaemia: correlation with high density lipoproteins. J Am Coll Cardiol 1996;27:1562-1570.

5. Goldbourt U, Yaari S, Medalie JH. Isolated low HDL cholesterol as a risk factor for coronary heart disease mortality. A 21-year follow-up of 8000 men. Arterioscl Thromb Vascul Biol 1997;17:107-113.

6. Nissen SE, Tsunoda T, Tuzcu EM, et al. Effect of recombinant ApoA-I Milano on coronary atherosclerosis in patients with acute coronary syndromes: a randomized controlled trial. JAMA 2003;290:2292-2300.

7. von Eckardstein A, Walter M, Holz H, Benninghoven A, Assmann G. Site-specific methionine sulfoxide formation is the structural basis of chromatographic heterogeneity of apolipoproteins A-I, C-II, and C-III. J Lipid Res 1991;32:1465-1476. 
8. Pagani F, Sidoli A, Giudici GA, Barenghi L, Vergani C, Baralle FE. Human apolipoprotein A-I gene promoter polymorphism: association with hyperalphalipoproteinemia. J Lipid Res 1990;31:1371-1377.

9. Civeira F, Pocovi M, Cenarro A, Garces C, Ordovas JM. Adenine for guanine substitution -78 base pairs 5' to the apolipoprotein (APO) A-I gene: relation with high density lipoprotein cholesterol and APO A-I concentrations. Clin Genet 1993;44:307-312.

10. Needham EW, Mattu RK, Rees A, Stocks J, Galton DJ. A polymorphism in the human apolipoprotein AI promoter region: a study in hypertriglyceridaemic patients. Hum Hered 1994;44:94-99.

11. Saha N, Tay JS, Low PS, Humphries SE. Guanidine to adenine (G/A) substitution in the promoter region of the apolipoprotein AI gene is associated with elevated serum apolipoprotein AI levels in Chinese non-smokers. Genet Epidemiol 1994;11:255-264.

12. Kamboh MI, Aston CE, Nestlerode CM, McAllister AE, Hamman RF. Haplotype analysis of two APOA-I/MspI polymorphisms in relation to plasma levels of apo A-I and HDL-cholesterol. Atherosclerosis 1996;127:255-262.

13. Wang XL, Liu SX, McCredie RM, Wilcken DE. Polymorphisms at the 5'-end of the apolipoprotein AI gene and severity of coronary artery disease. J Clin Invest 1996;98:372-377.

14. Meng QH, Pajukanta P, Valsta L, Aro A, Pietinen P, Tikkanen MJ. Influence of apolipoprotein A-I promoter polymorphism on lipid levels and responses to dietary change in Finnish adults. J Intern Med 1997;241:373-378.

15. Paul-Hayase H, Rosseneu M, Robinson D, Van Bervliet JP, Deslypere JP, Humphries SE. Polymorphisms in the apolipoprotein (apo) AI-CIII-AIV gene cluster: detection of genetic variation determining plasma apo AI, apo CIII and apo AIV concentrations. Hum Genet 1992;88:439-446. 
16. Sigurdsson G, Jr., Gudnason V, Sigurdsson G, Humphries SE. Interaction between a polymorphism of the apo A-I promoter region and smoking determines plasma levels of HDL and apo A-I. Arterioscler Thromb 1992;12:1017-1022.

17. Xu CF, Angelico F, Del Ben M, Humphries S. Role of genetic variation at the apo AICIII-AIV gene cluster in determining plasma apo AI levels in boys and girls. Genet Epidemiol 1993;10:113-122.

18. Angotti E, Mele E, Costanzo F, Avvedimento EV. A polymorphism (G-->A transition) in the -78 position of the apolipoprotein A-I promoter increases transcription efficiency. $\mathrm{J}$ Biol Chem 1994;269:17371-17374.

19. Kessling AM, Horsthemke B, Humohries SE. A study of DNA polymorphisms around the human apolipoprotein AI gene in hyperlipidaemic and normal individuals. Clin Genet 1985;28:296-306.

20. Wang XL, Badenhop R, Humphrey KE, Wilcken DE. C to T and/or G to A transitions are responsible for loss of a MspI restriction site at the 5'-end of the human apolipoprotein AI gene. Hum Genet 1995;95:473-474.

21. Wang XL, Badenhop R, Humphrey KE, Wilcken DE. New MspI polymorphism at +83 bp of the human apolipoprotein AI gene: association with increased circulating high density lipoprotein cholesterol levels. Genet Epidemiol 1996;13:1-10.

22. Zhang Q, Liu Y, Liu BW, Fan P, Cavanna J, Galton DJ. Common genetic variants of lipoprotein lipase and apolipoproteins AI-CIII that relate to coronary artery disease: a study in Chinese and European subjects. Molecular Genetics \& Metabolism 1998;64:177183.

23. Janus ED, for the Cardiovascular Risk Factor Prevalence Study Group. Hong Kong Cardiovascular Risk Factor Prevalence Study 1995-1996. Hong Kong, 1997:1-145. 
24. Stampfer MJ, Sacks FM, Salvini S, Willett WC, Hennekens CH. A prospective study of cholesterol, apolipoproteins, and the risk of myocardial infarction. $\mathrm{N}$ Engl $\mathrm{J}$ Med $1991 ; 325: 373-381$.

25. Badimon JJ, Fuster V, Badimon L. Role of high density lipoproteins in the regression of atherosclerosis. Circulation 1992;86:III86-94.

26. Steinmetz J, Boerwinkle E, Gueguen R, Visvikis S, Henny J, Siest G. Multivariate genetic analysis of high density lipoprotein particles. Atherosclerosis 1992;92:219-227.

27. Norum RA, Lakier JB, Goldstein S, et al. Familial deficiency of apolipoproteins A-I and C-III and precocious coronary-artery disease. N Engl J Med 1982;306:1513-1519.

28. Wu JH, Wen MS, Lo SK, Wu D. DNA polymorphisms of apolipoprotein B in the population of Taiwan. Journal of the Formosan Medical Association 1993;92:330-335.

29. Smith JD, Brinton EA, Breslow JL. Polymorphism in the human apolipoprotein A-I gene promoter region. Association of the minor allele with decreased production rate in vivo and promoter activity in vitro. J Clin Invest 1992;89:1796-1800.

30. Hegele RA, Brunt JH, Connelly PW. Genetic and biochemical factors associated with variation in blood pressure in a genetic isolate. Hypertension 1996;27:308-312.

31. Wu DA, Bu X, Warden $\mathrm{CH}$, et al. Quantitative trait locus mapping of human blood pressure to a genetic region at or near the lipoprotein lipase gene locus on chromosome 8p22. J Clin Invest 1996;97:2111-2118.

32. Ma YQ, Thomas GN, Critchley JA, Lee ZS, Chan JC, Tomlinson B. Association of the D8S282 marker near the lipoprotein lipase gene locus with systolic blood pressure in healthy Chinese subjects. J Hypertens 2002;20:2199-2204.

33. Pujia A, Gnasso A, Siclari D, et al. SstI RFLP and hypertension as risk factors for extracoronary atherosclerosis in a male population of southern Italy. Artery 1994;21:208221. 
Table 1: Clinical biochemical and genetic characteristics of the 271 non-diabetic Chinese control and hypertensive subjects

\begin{tabular}{|c|c|c|}
\hline & Controls (group A) & Hypertensive (group B) \\
\hline Numbers & 167 & 104 \\
\hline Age (years) & $36 \pm 11$ & $47 \pm 10$ \\
\hline Gender (\% male) & 36.8 & 47.1 \\
\hline Fasting glucose (mmol/L) & $4.9 \pm 0.4$ & $5.2 \pm 0.5$ \\
\hline Cholesterol (mmol/L) & $4.5 \pm 0.8$ & $5.5 \pm 1.3$ \\
\hline HDL-cholesterol (mmol/L) & $1.48 \pm 0.39$ & $1.26 \pm 0.32$ \\
\hline LDL-cholesterol (mmol/L) & $2.7 \pm 0.7$ & $3.7 \pm 1.3$ \\
\hline Triglyceride (mmol/L) & $0.68(0.63-0.73)$ & $1.19(1.10-1.29)$ \\
\hline Systolic blood pressure (mm Hg) & $111 \pm 9$ & $157 \pm 15$ \\
\hline Diastolic blood pressure (mm Hg) & $64 \pm 9$ & $94 \pm 11$ \\
\hline Mean arterial pressure (mm Hg) & $80 \pm 8$ & $115 \pm 10$ \\
\hline Body mass index $\left(\mathrm{kg} / \mathrm{m}^{2}\right)$ & $21.1 \pm 2.1$ & $26.2 \pm 4.1$ \\
\hline Waist circumference $(\mathrm{cm})$ & $69.2 \pm 6.3$ & $85.6 \pm 9.7$ \\
\hline C83T genotype (CC/CT, \%) & $89.8 / 10.2$ & $98.1 / 1.9$ \\
\hline C83T allele (C/T, \%) & $94.9 / 5.1$ & $99.9 / 1.0$ \\
\hline G-75A genotype (GG/GA/AA, \%) & 47.3 / 43.1 / 9.6 & 53.8 / 36.3 / 6.7 \\
\hline G-75A allele (G/A, \%) & $68.9 / 31.1$ & $73.6 / 26.4$ \\
\hline \multicolumn{3}{|c|}{ Mean \pm SD, geometric mean (geometric 95\% CI); all comparisons $\mathrm{p}<0.001$, except the ApoA- } \\
\hline I C83T genotype $(\mathrm{p}=0.012)$ or allele & $\mathrm{p}=0.011$ ) frequencies. & significant differences were \\
\hline
\end{tabular}


Table 2: Association between the apoA-I C83T polymorphism genotypes and lipid (HDL-cholesterol and triglycerides), and blood pressure parameters

\begin{tabular}{lccc}
\hline & \multicolumn{2}{c}{ ApoA-I C83T polymorphism genotypes } & P value \\
\cline { 2 - 3 } & CC (n=252) & CT (n=19) & \\
\hline HDL-cholesterol (mmol/L) & $1.39 \pm 0.38$ & $1.59 \pm 0.46$ & 0.029 \\
Triglyceride (mmol/L) & $0.86(0.80-0.91)$ & $0.61(0.52-0.72)$ & $<0.001$ \\
Systolic blood pressure (mm Hg) & $126 \pm 25$ & $115 \pm 15$ & 0.001 \\
Diastolic blood pressure (mm Hg) & $76 \pm 17$ & $68 \pm 11$ & 0.008 \\
Mean arterial pressure (mm Hg) & $94 \pm 19$ & $84 \pm 11$ & 0.002 \\
\hline
\end{tabular}

Mean \pm SD, geometric mean (geometric 95\% CI) 